\title{
Stephan Lessenich
}

\section{Zur Lage der bürgerlichen Demokratie in Deutschland}

"Möchten doch angesichts dessen diejenigen, welche in steter Angst davor leben,
es könnte in Zukunft in der Welt zu viel, Demokratie' und ,Individualismus' ge-
ben ..., sich endlich beruhigen: es ist, nur allzusehr, dafür gesorgt, daß die Bäume
des demokratischen Individualismus nicht bis in den Himmel wachsen."
Max Weber, Zur Lage der bürgerlichen Demokratie in Rußland (1906)

In der langen Geschichte der ,sozialen Frage" und ihrer sozialpolitischen Regulierung wird derzeit ein neues Kapitel aufgeschlagen. ${ }^{1}$ Bis in die jüngste Vergangenheit hinein wurde diese Geschichte durch wechselnde Diagnosen defizitärer Lebenslagen und -verhältnisse vorangetrieben: Gemeinsamer Nenner aller im Zuge des industriegesellschaftlichen Zeitalters thematisierten sozialen Fragen vom Pauperismus bis zu Heiner Geißler - war die Vorstellung, dass es den jeweils entdeckten sozialen Problemgruppen „schlecht" oder jedenfalls, gemessen an absoluten Größen oder vergleichbaren Gruppen, zu schlecht gehe. Der sozialpolitischen Programmatik unserer Tage hingegen liegt das entgegen gesetzte Deutungsmuster zugrunde: Den Menschen, so heißt es, gehe es "gut" - gewissermaßen zu gut. Es geht (in) der deutschen Sozialpolitik nicht länger um Defizite, sondern um Redundanzen. Was vorrangig politische Aktivitäten auslöst, sind nicht soziale Missstände, sondern soziale Besitzstände - sozialpolitische Überhänge aus vergangenen, „,besseren“ Zeiten.

1 Die zentrale These des vorliegenden Beitrages beruht auf einem Referat, das im November 2003 anlässlich einer Impulstagung zum Thema "Gibt es eine neue soziale Frage?" am Hamburger Institut für Sozialforschung gehalten wurde. Ich danke Heinz Bude und Berthold Vogel als Organisatoren sowie allen Teilnehmerinnen und Teilnehmern der Veranstaltung für die ebenso kontroverse wie konstruktive Diskussion desselben.
Das ,goldene Zeitalter" des Wohlfahrtsstaates ist vergangen. Die Tage stetigen Wirtschaftswachstums, dauerbeschäftigter Familienernährer und sorgloser Sozialrentner sind vorbei. Die Frage des nun anbrechenden "silver age" (vgl. TaylorGooby 2002) - des Zeitalters unkalkulierbarer Weltmärkte, brüchiger Erwerbsverläufe und erodierender Alterspyramiden - lautet: Wie sollen und können Wohlfahrtsverluste in der Wohlstandsgesellschaft verteilt und vermittelt werden? Dass diese Frage nicht nur eine materielle, sondern auch eine ideelle Dimension hat, also eine Frage politischer Steuerung (verteilen) und politischer Kommunikation (vermitteln) gleichermaßen ist, liegt auf der Hand. Aber wie wird diese doppelte sozial-politische Aufgabe bewältigt? In welcher Weise vollzieht sich die materielle und ideelle Umorientierung von Sozialpolitik im "silbernen Zeitalter"? Offenbar nach dem Prinzip kommunizierender Röhren: In dem Maße, wie dem „neuen“ Sozialstaat der sozialpolitische Verteilungsspielraum ökonomisch abhanden kommt bzw. politisch genommen wird, wächst das öffentlich zur Schau getragene Ressentiment gegen jene Demokratisierung sozialer Sicherheit und individueller Wohlfahrt, für die der ,alte" Sozialstaat - was auch immer man ansonsten gegen ihn sagen mag - in dem vergangenen halben Jahrhundert seiner Existenz durchaus gesorgt hat. Mit der unangenchmen Situation (oder gegebenenfalls auch nur: der Perspektive) konfrontiert, "den Gürtel enger schnallen" zu müssen, findet der deutsche Median- 
bürger Halt in der Gewissheit, dass die überkommenen "Autonomiegewinne der Leute" (Vobruba 2003) - Autonomiegewinne, wie sie im Verlaufe des 20. Jahrhunderts aus der Universalisierung des Staatsbürgerstatus und dessen beständiger Anreicherung mit sozialen Rechtsansprüchen hervorgegangen sind, ${ }^{2}$ - nun politisch beschnitten und auf ein „vernünftiges Maß" zurückgeführt werden.

Diese Gewissheit - und mit ihr eine gewisse Genugtuung, dass der akkumulierte Überschuss an sozialpolitisch unterfütterten Freiheiten zurückgefahren wird, gründet sich auf einen politischen „Reform "-Diskurs, den man in doppelter Hinsicht als freiheitskritisch bezeichnen könnte. Auf der einen Seite wird Freiheit hier nur mehr auf die eigenverantwortliche Nutzung der individuellen Marktchance reduziert (und sozialpolitische Intervention auf die Ermöglichung dieser Nutzung konzentriert). Auf der anderen Seite nimmt dieser Diskurs die - tatsächlich oder vermeintlich - gewachsene gesellschaftliche Sensibilität für (vermutete) Formen des sozial inkorrekten, d.h. gemeinschaftsschädigenden, Freiheitsgebrauchs in sich auf. Dabei stehen gesellschaftliches Ressentiment und politische Intervention in einem wechselseitigen Steigerungsverhältnis zueinander: Der viel und gern beschworene (und kritisierte) „Sozialneid“ der Mittelschichten der freilich weniger nach ,oben“ als vielmehr nach „unten ${ }^{6}$ und nach „nebenan" gerichtet ist: gegen das unverdiente, unwürdige Glück der Anderen, von den faulen Arbeitslosen über die unsozialen Kinderlosen bis zu den überversorgten Rentnern - wird im Prozess der „Modernisierung“ des Sozialstaats, der „Neujustierung von Rechten und Pflichten “ und der "Neubalancierung von Eigenverantwortung und Gemeinwohl", durch die politischen Eliten aufgenommen, reproduziert und verstärkt. Beispie-

2 So die klassische Theorie wohlfahrtsstaatlicher Entwicklung Marshalls (Marshall 1963). le gefällig? Nichts leichter als das: Willkommen im aktuellen Feindbildkabinett der deutschen Sozialpolitik.

\section{Sozialfiguren des Sozialneids}

Geraume Zeit schon, spätestens seit Beginn der langen Welle wohlfahrtsstaatlicher "Krisen" in den frühen 1970er Jahren, gehört die Figur des faulen Arbeitslosen zu den Hauptdarstellern der zyklisch wiederkehrenden Aufführungen des sozialpolitischen „Missbrauchs"-Dramas. So vorhersehbar sind die politischen Konjunkturen der Faulheitsdebatte, dass man fast von ,einem politischen Pawlow-Reflex“ (Oschmiansky et al 2003: 3) sprechen könnte: Gilt es Bundes- oder Landtagswahlen zu bestehen oder - man kann es ja mal versuchen - haltlose Versprechungen zur Halbierung oder ähnlich massiven Reduzierung der Arbeitslosenzahlen zu revidieren, kommt ein Hinweis auf die mangelnde Arbeitsbereitschaft der Erwerbslosen, verbunden mit der Forderung nach gebührendem Schutz der Steuer- und Beitragszahlergemeinschaft vor missbräuchlicher Inanspruchnahme ihrer Zahlungsbereitschaft, immer gut. „Wer arbeiten kann, aber nicht will, der kann nicht mit Solidarität rechnen": Gerhard Schröders geradezu klassische Adaptation des gesunden Volksempfindens für den politischen Demagogiegebrauch läutete im April 2001 die bislang letzte Runde in der langen Geschichte verschärfter Zumutbarkeitskriterien für arbeitslose Leistungsempfänger ein.

Das Praktische an der politisch-medialen Suggestion, derzufolge „vicle" Arbeitslose gar nicht arbeiten wollen, ist, dass sie nicht nur gegen anderslautende empirische Evidenz (vgl. z.B. Gebauer, Petschauer, Vobruba 2002; Gangl 1998) immun ist, sondern im Grunde genommen auch gar nicht empirisch widerlegt werden kann: Erst wenn der (die) letzte Arbeitslose die Statistik in Richtung Arbeitsmarkt verlassen hätte, wäre der Generalverdacht wirklich ausgeräumt. Inso- 
fern wird uns der entsprechende Kampagnenzyklus auf unabsehbare Zeit crhalten bleiben: Die Pflege des Wahnbildes vom faulen Arbeitslosen ist wahlpolitisch wie medienökonomisch rational, und die Skandalisierung devianter Existenzformen wirkt zudem - in einer Mittelschichtsgesellschaft, deren Wohlstand zunehmend prekär zu werden droht, sozial stabilisierend (vgl. dazu auch Lessenich 2004: 38ff). Doch dies ist noch nicht alles. Die Remoralisierung der Arbeitslosigkeit, die in der Figur des faulen Arbeitslosen aufscheint, und die damit verbundene Forderung, die Erwerbslosen generell, wahlweise durch Sanktionsoder durch Anreizinstrumente, zur aktiven Wiedererlangung ihrer Beschäftigungsfähigkeit anzuhalten, greift in ihren Effekten über die Gruppe der unmittelbar Betroffenen hinaus. Auf paradoxe Weise wendet sich die aus dem mehrheitsgesellschaftlichen Ressentiment gegen eine sozialstaatlich geschützte Minderheit geborene, politisch inszenierte Verpflichtungs- und Disziplinierungskampagne gegen die sozialstaatlichen Statusverbürgungen der Mehrheit selbst. Die sozialwissenschaftliche Legitimation dieser expansiven, tendenziell grenzenlosen Dynamik des veränderten sozialpolitischen Umgangs mit der arbeitslosen Minderheit wird interessanterweise von einer Gruppe von Arbeitsmarktforschern geliefert, die sich kritisch mit dem "Missbrauch des Missbrauchverdachts" (Vobruba 1989: 186-188) auseinandersetzen. Um die Arbeitslosen, die ,gegenüber der Solidargemeinschaft in der Pflicht stehen, ... intensiv nach Erwerbschancen zu suchen und diese auch wahrzunehmen", vor falschen Verdächtigungen und diskriminierenden Vornahmen zu bewahren, gelte es - so das Argument -, die entsprechenden arbeitsmarktpolitischen Verhaltensanforderungen („die Erweiterung des Entscheidungsspielraums auf individueller Ebene") für alle Erwerbspersonen verbindlich zu machen. Die politische Logik des Gedankens ist bestechend: „Die präventive In- klusion der ,Insider' durch Verpflichtung bzw. durch Anreize zur alltäglichen Vorsorge und Verbesserung der Beschäftigungsfähigkeit würde der reaktiven Verpflichtung der Arbeitslosen zur Arbeitsund Mobilitätsbereitschaft nicht nur mehr Legitimität, sondern auch mehr Wirlksamkeit verleihen." (Oschmiansky et al. 2003: 29). Ja, dem wird wohl so sein: Altägliche Marktfähigkeitsvorsorge stellt nicht als exklusives Minderheitenerfordernis, sondern als allgemeinverbindliches Inklusionsgebot eine sozialpolitisch reizvolle Programmoption dar. Oder anders formuliert: Sind wir nicht alle ein bisschen faule Arbeitslose?

Der Fall der unsozialen Kinderlosen ist etwas anders gelagert. Ihre Entdeckung für die bundesdeutsche sozialpolitische Debatte beginnt im Grunde erst mit der öffentlichen Bewusstwerdung der drohenden "demographischen Katastrophe". Seither ist Adenauers auf die Sicherheit der umlagefinanzierten Rente gemünztes Diktum „Kinder kriegen die Leute sowieso" - so die Überlieferung - rasch zum geflügelten Unwort der deutschen Sozialpolitikgeschichte aufgestiegen, und das Bundesverfassungsgericht hat sich zum obersten Fürsprecher der Leistungsgemeinschaft der Kindererziehenden aufgeschwungen. Immer wieder, vom Trümmerfrauen- bis zum "Beitragskinder"Urteil, wurde von höchstrichterlicher Seite die sozialpolitische Honorierung des generativen Beitrags zur Sicherung der sozialen Sicherung eingeklagt. Zuletzt forderten die Verfassungshüter, Erziehende seien in der Pflegeversicherung - und zukünftig womöglich auch in den anderen Sozialversicherungszweigen - relativ zu nicht-erziehenden Versicherten zu entlasten. Bezeichnenderweise verstand die Bundesregierung dies als Aufforderung zur Belastung der Kinderlosen (mit einem pauschalen monatlichen $\mathrm{Zu}$ satzbeitrag) und erklärte - in einem Anfall sozialpolitischer Kreativität - Eltern ohne Kinder mit Kindergeldanspruch sogleich zu Kinderlosen ehrenhalber, denen der Solidarbeitrag zugunsten der 
„richtigen“, aktiv erziehenden Eltern gleichfalls zuzumuten sei. Der "Wert“ eines Kindes, den findige Ölonomen für die Rentenversicherung bereits berechnet haben (Sinn 1997), hält somit - auch dies ein Paradoxon sozialpolitischer Intervention - in der Logik der Pflegeversicherungsreform nicht lange vor. ${ }^{3}$

Umso mehr und nachhaltiger streiten besagte Ökonomen, im Einklang mit dem „familienfreundlichen “ Zeitgeist, für die sozialpolitische Diskriminierung jener Personen, die die wohlfahrtsstaatliche Sozialisierung des Altersrisikos nutzen, um auf Nachwuchs zu verzichten und sich von den Kindern anderer Leute aushalten zu lassen. Besonders altiv in diesem Sinne ist Hans-Werner Sinn, Präsident des Münchner Wirtschaftsforschungsinstituts "ifo", der - wenn er nicht gerade für die Rückkehr zum "Heimatlandprinzip“ wirbt, um sozialpolitische Wanderungsanreize in der erweiterten EU zu neutralisieren (vgl. Sinn 2000, dazu auch Lessenich 2004: 37f), für die "Rente nach Kinderzahl“" mobilisiert. Die Rentenversicherung, so Sinns Argument (vgl. zum Folgenden Sinn 2003), sei im Kern „eine Versicherung gegen Kinderlosigkeit" und habe damit „Fehlanreize für die Familienplanung" institutionalisiert. Jede Generation müsse zum Zweck ihrer Alterssicherung entweder Human- oder Realkapital bilden, d.h. Kinder in die Welt setzen und aufziehen oder aber einen Kapitalstock aufbauen, von dem sie dereinst zehren könne. Wer mithin freiwillig auf Humankapitalproduktion verzichte, müsse „die Konsequenzen tragen und selbst auf dem Wege

3 Wie im Übrigen auch die Pflegeversicherungsreform selbst: Nach neuesten Informationen (vgl. „Schröder setzt Reform der Pflege aus", Frankfurter Allgemeine Zeitung rom 29.1.2004, S. 13) soll nun statt des geplanten Beitragszuschlags für die weit definierten „Kinderlosen" doch eine Entlastung der Erziehenden (in Form von Freibeträgen auf das beitragspflichtige Einkommen) erfolgen, wobei auch diese Regelung aber nur Personen mit Anspruch auf Kindergeld zugute kommen soll. der Ersparnis für Ersatz sorgen“, anstatt von den Reproduktionsleistungen Dritter zu profitieren. ,Statt eine ganze Generation kollektiv in dic Verantwortung zu nehmen, sollten die notwendigen Rentenkürzungen und das kompensierende Riester-Sparen auf die Kinderlosen konzentriert werden." Bei entsprechender Belastung der Übeltäter - Halbierung ihrer Umlagerenten, Verpflichtung zur Verwendung von acht Prozent des Bruttoeinkommens für die ergänzende private Vorsorge - werde es mit einiger Wahrscheinlichkeit zu gesellschaftlich wünschenswerten Verhaltensänderungen kommen: „Manch ein bislang unschlüssiges Paar wird sich unter diesen Umständen vielleicht doch für Kinder entscheiden. Alle Erfahrung zeigt, dass gerade auch die Familienplanung sehr stark auf ökonomische Anreize reagiert." Mit anderen Worten: Wer nicht zeugen will, muss fühlen. Oder das Ganze einfach noch $\mathrm{mal}$ in Ruhe durchrechnen. ${ }^{4}$

Aber nicht nur die unverantwortlichen Kinderlosen, auch die überversorgten Rentner werden allenthalben - und gleichsam spiegelbildlich - der Ausbeutung der Solidargemeinschaft bezichtigt. Die Ansicht, dass "die Jungen" die Zeche eines sorgenfreien Lebens „der

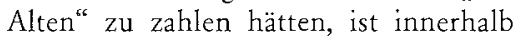
kürzester Zeit zum kaum mehr widerspruchsfähigen Standardargument der sozialpolitischen Debatte geworden, und wer öffentlich als Rächer der von den

4 Es gehört zu den Lebenslügen der liberâlen Ökonomik, entsprechende - durch politische Intervention erzwungene - Verhaltensänderungen $z u$ Wirlkungseffekten von „Entstaatlichung“ zu erklären: „Das heißt nicht, dass einer staatlichen Bevölkerungspolitik das Wort geredet werden soll, deren. Ziel es ist, in die freien Entscheidungen der Menschen einzugreifen und sie bei der Kinderwahl zu bevormunden, im Gegenteil. ... Die Rente nach der Kinderzahl einzuführen heißt, den Grad der Sozialisierung zurïckzufahren, also den Staat wieder ein Stück weit aus der Familienplanung herauszunehmen " (Sinn 2003). Das nennt man wohl neoklassische Dialektik. 
Wirtschaftswundergewinnlern Enterbten auftritt und in bemüht-besorgter $\mathrm{Ma}$ nier "Generationengerechtigkeit" einklagt, erntet den wohlfeilen Applaus und das gedankenlose Kopfnicken des Publikums. In diesem gesellschaftlichen Klima können historische Errungenschaften der Alterssicherungspolitik wie z.B. die Idee der Teilhabe der älteren Generation am gesellschaftlichen Reichtum ihrer Zeit ohne großes Aufsehen zur Disposition gestellt werden. Was die Sozialhilfeklientel (von den Asylsuchenden ganz zu schweigen) schon seit längerem kennt - die Abkopplung ihres Sicherungsniveaus rom Lebensstandard der Erwerbstätigen - wird mit den jüngsten Sozialreformen nun endgültig auch dem durchschnittlichen Rentnerhaushalt verordnet. Dass dieser materiell überausgestattet sei und cine Reduktion seiner Lohnersatzansprüche daher durchaus angemessen erscheint, wird dabei stillschweigend unterstellt (wenn. nicht sogar explizit hervorgehoben).

Was im Bild von den gierigen Alten und in der - in allen politischen Lagern angestimmten - Klage über eine Rentenpolitik „auf Kosten künftiger Generationen“ aufscheint, ist nichts weniger als eine Revision des überkommenen gesellschaftlichen "Generationenvertrags". Beruhte dieser auf der Idee der Umverteilung des in der laufenden Periode erwirtschafteten Sozialproduktes zum Zweck der Existenzsicherung der jeweiligen Rentnerpopulation, so werden nunmehr den gegenwärtigen Rentenbeziehern jene materiellen Einschränkungen auferlegt, welche die heutigen Erwerbstätigen in Zukunft auf sich selbst zukommen sehen. Dieses verquere, ahistorische Verständnis von "Generationengerechtigkeit" kann als prototypisches Beispiel sozialpolitisch instrumentalisierten Sozialneides gelten - und seiner paradoxen Implikationen: Wird hier doch eine gesellschaftliche Generation, die im Übrigen ihrer Verpflichtung zur Humankapitalproduktion recht gewissenhaft nachgekommen ist, gewissermaßen präventiv für eine zukünftig erwartete Verringerung des gesellschaftlichen Umverteilungsspieiraums haftbar gemacht. Man könnte auch sagen: Für den Schutz des ungeborenen Beitragszahlerlebens geht die deutsche Sozialpolitik schon heute über Rentenanspruchsleichen.

\section{Die Ängste der Wohistands- gesellschaft}

Faule Arbeitslose, unsoziale Kinderlose, überversorgte Rentner - die Feindbildproduktion der deutschen „Sozialreform" gebiert fast schon täglich neue Monster. Und ob es nun die Idee der Arbeitsdienstverpflichtung, des Familienwahlrechts oder eines "GenerationenTÜVs" ist: Keiner der auf die Sozialfiguren des Sozialneids gemünzten politischen Reformvorschläge scheint zu autoritär, zu reaktionär oder - seien wir ehrlich - zu blöde zu sein, um nicht doch irgendwie Gehör zu finden. Die "Schluss mit lustig"-Stimmung im Lande macht fast alles möglich - oder zumindest schon mal denkmöglich. Den letztlich kontingenten Objekten gesellschaftlicher Sozialneiddiskurse - die keineswegs bloß auf "Randgruppen“" zielen, sondern selbst ,in der Mitte der Gesellschaft" solidaritätsausbeuterische Sozialpraktiken ausmachen, denen es Einhalt zu bieten gelte, - stehen mithin harte Zeiten bevor. Der Ratsvorsitzende der Evangelischen Kirche in Deutschland (EKD), Bischof Huber, predigte entsprechend in seinem Neujahrsgottesdienst Verzicht und forderte zugleich, mehr vor- denn fürsorglich, dazu auf, „schärfere soziale Gegensätze in unserem Land" auszuhalten (Huber 2004: 1). Die katholischen Bischöfe ihrerseits mahnen in einem praktisch zeitgleich veröffentlichten "Impulstext" Initiativen an, „das Soziale neu [zu] denken", und gehen sogleich mit gutem Beispiel voran, indem sie dafür plädieren, „die Lebenslagen und -risiken enger zu definieren, für die eine Sicherung notwendig ist" (Die deutschen Bischöfe 2003: 17). Da soll noch einmal jemand 
sagen, die beiden großen Kirchen gingen nicht mit der Zeit. ${ }^{5}$

Was in dieser Zeit, der Zeit wohlfeiler Sozialstaatskritik, verloren geht, ist der Sinn dafür, dass der moderne Wohlfahrtsstaat jene Formen der Selbstorganisation und Armenhilfe, die den heutigen Kritikern als allein legitimer Kern sozialpolitischer Intervention gelten, gerade zu überwinden trachtete und dass es nicht der kostenbewusste Appell an "Eigenverantwortung" und die öffentliche Selbstbeschränkung auf Hilfen für die ,wirklich Bedürftigen" sind, die dieses wohlfahrtsstaatliche Arrangement legitimatorisch begründen oder begründen können. Was den entwickelten Wohlfahrtsstaat „zusammenhält “ und allein zusammenhalten kann ist ein normatives Fundament verallgemeinerter Gegenseitigkeit, die gesellschaftsweite Durchsetzung des demokratischen Prinzips wechselseitiger Anerkennung und Unterstützung. Dieses Prinzip, das rein denklogisch die Solidarität auch mit den vermeintlich Faulen, Unsozialen und Überversorgten einschließt, wird durch die unablässige politische Konstrulktion, Moralisierung und Skandalisierung eben dieser Sozialfiguren effektiv ausgehöhlt. Die politische Apologie des Sozialneids gräbt beständig an den sozialmoralischen Fundamenten eines universalistischen Sozialstaats. Dieses moralökonomische Argument aber taucht mittlerweile nirgendwo mehr auf,

5 Ganz im Gegenteil: Bischof Huber (2004) liegt mit der Aussage, Wir haben soziale Errungenschaften einzuschränken, wenn wir sie erhalten sollen.", voll im Trend des gegenwärtigen sozialpolitischen Diskurses, und die Denkschrift der Deutschen Bischofskonferenz reproduziert von vorne bis hinten so ziemlich alle Gemeinplätze der aktuellen Sozialstaatsdebatte, von „Reformen sind notwendig. Deutschland verträgt keinen weiteren Stillstand." (Die deutschen Bischöfe 2003: 3) über "Heute erscheint nicht mehr vorrangig die Verteilungsgerechtigkeit als das Hauptproblem.“ (ebd.: 16) bis hin zu „Durchbrochen werden muss der Vorrang ... der Interessen von heute vor den Interessen von morgen" (ebd.: 28). Das ist gelebte Ölkumene. auch in den sozialpolitischen Kirchenmännerrechnungen nicht - dabei müssten gerade sie es besser wissen: It's the moral economy, stupid!

Stattdessen erleben wir die Wiederholung der immergleichen Kampagnen, die offenbar einer uralten - und beliebig erneuerbaren - Angst vor „zu viel“ Individualität und Autonomie, ja vor ,zu viel“" Demokratie im Sinne eines demokratischen Wohlfahrtsindividualismus entspringen und diese Ängste wiederum bedienen. Es ist ziemlich genau hundert Jahre her, dass Max Weber, mit Blick auf die „Lage der bürgerlichen Demokratie in Rußland“, „diejenigen, welche in steter Angst davor leben, es könnte in Zukunft in der Welt zu viel ,Demokratie' und ,Individualismus' geben", zu mahnen versuchte, sie möchten sich doch „endlich beruhigen: es ist, nur allzusehr, dafür gesorgt, daß die Bäume des demokratischen Individualismus nicht bis in den Himmel wachsen. ... alle ökonomischen Wetterzeichen", so Weber damals weiter, wiesen vielmehr ,nach der Richtung zunehmender ,Unfreiheit" "Weber 1988: 63). Nun: Es hat offensichtlich nichts genutzt. Heute, unter Bedingungen ausgebauter Wohlfahrtsstaatlichkeit, kehrt die von Weber inkriminierte Sorge als Angst vor „zu viel" Wohlfahrtsdemokratie und vor „zu viel" Wohlfahrtsindivdualismus, als Angst vor jenen Freiheitsgewinnen der Wohlfahrtsgesellschaft, die der gesellschaftlich verallgemeinerten Solidarität entspringen, zurück - und wird mit der politischen Anerkennung der heilenden, "charakterbildenden Kraft" (Streeck 1998: 42) von Marktzwängen und der autoritativen Durchsetzung von Gemeinwohlkonstruktionen gegen öffentlich enttarnte Solidaritätsgewinnler beantwortet. Insofern weisen heute in Deutschland nicht nur die ökonomischen, sondern auch die politischen Wetterzeichen ,nach der Richtung zunehmender ,Unfreiheit" ". Wir lernen: Max Weber hatte - wie fast immer recht. Und die Geschichte der „sozialen Frage" geht weiter. 


\section{Literatur}

Die deutschen Bischöfe - Kommission für gesellschaftliche und soziale Fragen (2003): Das Soziale neu denken. Für eine langfristig angelegte Reformpolitik, Bonn, 12. Dezember 2003

Gangl, Markus (1998): Sozialhilfebezug und Arbeitsmarktverhalten. Eine Längsschnittanalyse der Übergänge aus der Sozialhilfe in den Arbeitsmarkt, in: Zeitschriff fur Soziologie 27 (3), S. 212-232.

Gebauer, Ronald; Petschauer, Hanna; Vobruba, Georg (2002): Wer sitzt in der Armutsfalle? Selbstbehauptung zwischen Sozialhilfe und Arbeitsmarkt, Berlin: edition sigma.

Huber, Wolfgang (2004):'Einen kleiner werdenden Kuchen fair verteilen', Frankfurter Allgemeine Zeitung vom 2.1.2004.

Lessenich, Stephan (2004): Auf welcher Baustelle wollen wir leben? Die ,Krise' des Wohlfahrtsstaats, die ,Reform' der Sozialpolitik und die Chancen soziologischer Diagnose, in: Soziologische Revue 27 (1), S. $29-43$

Marshall, Thomas H. (1963): Citizenship and Social Class, in: ders., Sociology at the crossroads and other essays, London: Heinemann, [zuerst 1949], S. 67-127.

Oschmiansky, Frank; Schmid, Günther; Kull, Silke (2003): Faule Arbeitslose? Politische Konjunkturen und Strukturprobleme der Missbrauchsdebatte, in: Leviathan 31 (1), S. 3-31

Sinn, Hans-Werner (1997 ): The Value of Children and Immigrants in a Pay-As-YouGo Pension System: A Proposal for a Partial Transition to a Funded System, NBER
Working Paper 6229, National Bureau of Economic Reserach, Cambridge/Mass.

Sinn, Hans-Werner (2000): Die Osterweiterung der EU und die Zukunft des Sozialstaates, in: Stephan Leibfried und Uwe Wagschal (Hg.), Der deutsche Sozialstaat. Bilanzen Reformen - Perspektiven, Frankfurt/New York: Campus, S. 474-489.

Sinn, Hans-Werner (2003): Rente nach Kinderzahl, in: Frankfurter Allgemeine Zeitung vom 14.1.2003, S. 12.

Streeck, Wolfgang (1998): Einleitung: Internationale Wirtschaft, nationale Demokratie?, in: ders. (Hg.), Internationale Wirtschaft, nationale Demokratie. Herausforderungen für die Demokratietheorie, Frankfurt/New York: Campus, S. 11.58.

Taylor-Gooby, Peter (2002): The Silver Age of the Welfare State: Perspectives on Resilience, in: Journal of Social Policy 31 (4), S. 597-621.

Vobruba, Georg (1989): „Sozialstaatskritik. Neokonservative Interpretationen zur Vereinzelung im Wohlfahrtsstaat", in: ders., Arbeiten und Essen. Politik an den Grenzen des Arbeitsmarkts, Wien: Passagen, S. 181-192

Vobruba, Georg (2003): Freiheit: Autonomiegewinne der Leute im Wohlfahrtsstaat, in: Stephan Lessenich ( $\mathrm{Hg}$.), Wohlfahrtsstaatliche Grundbegriffe. Historische und aktuel. le Diskurse, Frankfurt/New York: Campus, S. 137-155.

Weber, Max (1988): Zur Lage der bürgerlichen Demokratie in Russland, in: ders., Gesammelte Politische Schriften, Herausgegeben von Johannes Winckelmann, Tübingen: J.C.B. Mohr (Paul Siebeck), 5. Auf1. [zuerst 1906], S. 33-68. 